\title{
O RETRATO DOS EVENTOS ADVERSOS EM UMA CLÍNICA MÉDICA: ANÁLISE DE UMA DÉCADA
}

\author{
Natália Nunes Costa ${ }^{1}$, Ana Elisa Bauer de Camargo Silva ${ }^{2}$, Juliana Carvalho de Lima ${ }^{3}$, Mara Rubia de Sousa \\ Barbosa $^{4}$, Juliana Santana de Freitas ${ }^{5}$, Ana Lúcia Queiroz Bezerra ${ }^{2}$
}

RESUMO: Estudo observacional do tipo descritivo transversal que teve como objetivo analisar os eventos adversos ocorridos em unidade de clínica médica de um hospital de Goiás. A coleta de dados foi realizada a partir da leitura de 12 livros de registros de enfermagem preenchidos de janeiro de 2005 a dezembro de 2014. Foram encontrados 1360 eventos adversos, dos quais $408(30 \%)$ se referiam a retiradas acidentais de sondas, cateteres e cânulas; 288 (21,2\%) erros de medicação; 157 (11,5\%) erros envolvendo acesso vascular; 134 (9,8\%) casos de infecção relacionada à assistência à saúde; $115(8,4 \%)$ processos alérgicos; $91(6,7 \%)$ quedas de pacientes; 72 (5,3\%) úlceras por pressão; 56 (4,1\%) episódios de falta de hemoderivados e 39 (2,9\%) evasões. O estudo aponta o aumento dos registros dos eventos adversos ao longo da década, indicando a necessidade de adoção de medidas de prevenção e maior conscientização da enfermagem quanto à importância do relato desse incidente.

DESCRITORES: Cuidados de enfermagem; Erros médicos; Gestão de riscos; Prevenção de acidentes; Segurança do paciente.

\section{THE PORTRAIT ADVERSE EVENTS IN A MEDICAL CLINIC: ANALYSIS OF A DECADE}

\begin{abstract}
This was a cross-sectional descriptive observational study aimed at analyzing adverse events in a medical clinic unit of a hospital in Goiás. Data were collected from the reading of 12 nursing registry books completed from January 2005 to December 2014 The analysis found 1,360 adverse events, of which 408 (30\%) referred to accidental removal of probes, catheters, and cannulas; 288 $(21.2 \%)$ to medication errors; $157(11.5 \%)$ to errors involving vascular access; $134(9.8 \%)$ to cases of healthcare-associated infections; $115(8.4 \%)$ to allergic processes; $91(6.7 \%)$ to patient falls; $72(5.3 \%)$ to pressure ulcers; $56(4.1 \%)$ to episodes of lack of blood derivatives; and $39(2.9 \%)$ to evasions. The study shows an increase in the registry of adverse events over the decade, pointing out the need to adopt preventive measures and raising nursing personnel's awareness about the importance of reporting these incidents. DESCRIPTORS: Nursing care; Medical errors; Risk management; Accident prevention; Patient safety.
\end{abstract}

\section{DESCRIPCIÓN DE EVENTOS ADVERSOS EN UN SERVICIO DE CLÍNICA MÉDICA: ANÁLISIS DE UNA DÉCADA}

RESUMEN: Estudio observacional de tipo descriptivo transversal objetivando analizar los eventos adversos ocurridos en unidad de clínica médica de un hospital de Goiás. Datos recolectados a partir de lectura de 12 libros de registros de enfermería completados entre enero de 2005 y diciembre de 2014. Fueron hallados 1360 eventos adversos, de los cuales 408 (30\%) hacían referencia a retiros accidentales de sondas, catéteres y cánulas; 288 (21,2\%) errores de medicación; 157 (11,5\%) errores involucrando acceso vascular; $134(9,8 \%)$ casos de infección relacionada a atención de salud; 115 (8,4\%) procesos alérgicos; 91 (6,7\%) caídas de pacientes; 72 (5,3\%) úlceras por presión; 56 (4,1\%) episodios de falta de hemoderivados y $39(2,9 \%)$ evasiones. El estudio expresa el aumento de registros de eventos adversos a lo largo de la década, indicando necesidad de adopción de medidas preventivas y mayor concientización de los enfermeros respecto de la importancia de informar estos incidentes.

DESCRIPTORES: Atención de Enfermería; Errores Médicos; Gestión de Riesgos; Prevención de Accidentes; Seguridad del Paciente.

${ }^{1}$ Discente de Enfermagem, Universidade Federal de Goiás. Goiânia, GO, Brasil.

${ }^{2}$ Enfermeira. Doutora em Enfermagem. Docente de Enfermagem da Universidade Federal de Goiás. Goiânia, GO, Brasil.

${ }^{3}$ Enfermeira. Mestranda em Enfermagem. Universidade Federal de Goiás. Goiânia, GO, Brasil.

${ }^{4}$ Enfermeira. Especialista em Estratégia de Saúde da Família. Enfermeira da Unidade Básica de Saúde da Família Nova Olinda da Secretaria Municipal de Saúde de Aparecida de Goiânia. Aparecida de Goiânia, GO, Brasil.

${ }^{5}$ Enfermeira. Doutoranda em Enfermagem. Universidade Federal de Goiás. Goiânia, GO, Brasil.

Autor Correspondente:

Ana Elisa Bauer de Camargo Silva

Universidade Federal de Goiás

R. 227 Qd 68, S/N - 74605-080 - Goiânia, GO, Brasil

E-mail: anaelisabauer@gmail.com
Recebido: 01/03/2016

Finalizado: 15/07/2016 


\section{- INTRODUÇÃO}

O paciente, ao ser admitido em um hospital, deve receber atendimento seguro e de qualidade. Todavia, falhas na assistência à saúde vêm causando incidentes nos ambientes de cuidado e têm sido apontadas como um grave problema de saúde pública global.

A publicação do relatório do Institute of Medicine, denominado "To Err is Human: Building A Safer Health System" evidenciou que 1.000.000 de eventos adversos (EA) evitáveis ocorrem anualmente nos Estados Unidos da América, contribuindo para a morte de 44 a 98 mil pessoas em hospitais anualmente, o que situou os EA entre a quarta e nona causa mais importante de óbito ${ }^{(1)}$.

Estudo realizado na Itália avaliou 1501 registros clínicos no arquivo eletrônico de altas hospitalares e mostrou que $3,3 \%$ dos pacientes internados no hospital experimentaram pelo menos um $\mathrm{EA}^{(2)}$.

Estudo realizado no Brasil identificou a magnitude e gravidade dos EA ocorridos em clínica cirúrgica, sendo que dos 218 eventos relatados, $78 \%$ resultaram em dano leve, 16,5\% em dano moderado e 2,3\% em dano grave, sendo que 3,2\% resultaram em óbito ${ }^{(3)}$.

A análise retrospectiva de 826 boletins de notificação de EA de um hospital universitário do Estado de São Paulo indicou a ocorrência de 1219 EA, incidentes e ocorrências administrativas, sendo que $12,8 \%$ foram em unidade de Clínica Médica ${ }^{(4)}$.

Em relação ao local de ocorrência dos EA, estudo realizado em prontuários de três hospitais de ensino do estado do Rio de Janeiro, com uma amostra de 1103 pacientes, identificou que 56 sofreram EA evitáveis, sendo que $56,9 \%$ destes se deram no quarto ou na enfermaria ${ }^{(5)}$.

As consequências dos EA podem ser o prolongamento do tempo de internação, incapacidade temporária ou definitiva, até o óbito do paciente ${ }^{(6)}$.

Pesquisa conduzida na base de dados do National Service United Kingdom Health, identificou a ocorrência de 2010 EA fatais em um período de dezessete meses, sendo os mais comuns: falhas em agir ou reconhecer o dano causado ao paciente (23\%), quedas $(10 \%)$ e infecções relacionadas à assistência à saúde $(10 \%)^{(7)}$.

Perante esse contexto, os sistemas assistenciais devem ser melhorados e os profissionais devem estar atentos à realidade de sua instituição, identificando e analisando os eventos adversos, gerenciando riscos e, principalmente, planejando e aderindo às condutas necessárias para reduzir danos e aumentar a qualidade e segurança dos pacientes.

Ao conhecer os tipos de eventos ocorridos, espera-se que a enfermagem, bem como gestores e toda a equipe multiprofissional, atuem de forma visionária, assumindo uma posição de vanguarda na busca de ações que modifiquem esse panorama.

Considerando que a segurança do paciente e a redução de falhas nos cuidados de saúde devem ser preocupações prioritárias para instituições e profissionais de saúde, este estudo se propõe a analisar os eventos adversos de uma unidade de clínica médica de um hospital de ensino de Goiânia - Goiás, fornecendo assim, informações para o planejamento de estratégias de melhorias que aumentem a segurança do paciente.

\section{MÉTODO}

Estudo observacional do tipo descritivo transversal desenvolvido na unidade de clínica médica de um hospital de ensino de Goiânia. A clínica médica, atualmente, dispõe de 59 leitos, divididos em 13 enfermarias. A unidade é responsável pelo atendimento de pacientes adultos, das seguintes especialidades médicas: cardiologia, endocrinologia, gastroenterologia, hematologia, imunologia, nefrologia, oncologia, pneumologia e reumatologia, e, no ano de 2013, possuía uma média de 240 internações/mês ${ }^{(8)}$.

A coleta de dados foi realizada de janeiro a abril de 2015 e os dados foram obtidos dos livros utilizados 
pela enfermagem para realização de registros de passagem de plantão, comunicados internos, solicitações e anotações de intercorrências, entre janeiro de 2005 e dezembro de 2014. Contudo, no ano de 2012, apenas o período de janeiro a julho foi analisado, devido ao desaparecimento dos demais livros. Ao todo 12 livros, tipo ata, foram analisados, contendo 400 páginas cada.

A unidade de análise foi o registro de enfermagem. Procedeu-se a leitura das anotações, visando identificar os registros relacionados à ocorrência de EA que, quando detectados, eram transcritos na íntegra para um instrumento semiestruturado de coleta de dados, elaborado pelas pesquisadoras, composto de itens abertos e fechados, que contemplavam informações sobre o horário de ocorrência do evento, tipo de evento, consequências para o paciente, condutas adotadas, e categoria dos profissionais envolvidos, assim como a transcrição do registro identificado.

Os dados foram estruturados em planilha eletrônica do Microsoft Office Excel versão 2011 e analisados estatisticamente empregando-se o software Statistical Package for Social Sciences (SPSS) versão 19.0, apresentando as frequências absolutas e relativas. A incidência de EA foi calculada pela expressão: número de EA/número total de pacientes admitidos na clínica médica no período x $100^{(9)}$.

O estudo foi aprovado pelo Comitê de Ética em Pesquisa Médica Humana e Animal do Hospital das Clínicas da Universidade Federal de Goiás (Protocolo $N^{\circ}$ 064/2008) e desenvolvido de acordo com a Resolução 466/12 do Conselho Nacional de Saúde ${ }^{(10)}$.

\section{RESUlTADOS}

Um total de 13.314 admissões ocorreu na clínica médica entre janeiro de 2005 e dezembro de 2014. Neste período, foram identificados 1.360 eventos adversos. Assim, a incidência de EA por 100 pacientes admitidos foi 10,2 (1.360 de 13.314; IC 95\%: 9,7-10,7). A distribuição dos EA está apresentada na Tabela 1.

Observou-se na Tabela 1 que os eventos adversos mais frequentes foram a retirada acidental de sondas, cateteres e drenos $(408 ; 30 \%)$, seguida de erro de medicação $(288 ; 21,2 \%)$ e evento relacionado a acesso vascular (157; 11,5\%). Cabe ressaltar que em algumas anotações foram identificados mais de um EA.

A Figura 1 apresenta a série histórica do número de eventos adversos e da incidência ao longo do período analisado.

Tabela 1 - Distribuição dos eventos adversos ocorridos na unidade de clínica médica de um hospital de ensino, segundo tipo e ano de ocorrência. Goiânia, GO, Brasil, 2015

\begin{tabular}{|c|c|c|c|c|c|c|c|c|c|c|c|c|}
\hline \multirow{2}{*}{$\begin{array}{l}\text { Tipo de Evento } \\
\text { Adverso }\end{array}$} & \multicolumn{10}{|c|}{ Ano } & \multicolumn{2}{|c|}{ Total } \\
\hline & 2005 & 2006 & 2007 & 2008 & 2009 & 2010 & 2011 & 2012* & 2013 & 2014 & $\mathbf{N}$ & $\%$ \\
\hline $\begin{array}{l}\text { Retirada acidental de } \\
\text { sondas, cateteres e } \\
\text { drenos }\end{array}$ & 55 & 35 & 34 & 27 & 46 & 41 & 47 & 24 & 48 & 51 & 408 & 30 \\
\hline Erro de medicação & 20 & 5 & 12 & 11 & 11 & 22 & 49 & 25 & 71 & 62 & 288 & 21,2 \\
\hline $\begin{array}{l}\text { Evento relacionado a } \\
\text { acesso vascular }\end{array}$ & 18 & 8 & 13 & 5 & 5 & 19 & 32 & 12 & 16 & 29 & 157 & 11,5 \\
\hline $\begin{array}{l}\text { Infecção relacionada } \\
\text { à assistência à saúde }\end{array}$ & 20 & 11 & 4 & 5 & 8 & 26 & 26 & 14 & 18 & 2 & 134 & 9,8 \\
\hline Processo alérgico & 12 & 16 & 13 & 13 & 6 & 11 & 14 & 8 & 11 & 11 & 115 & 8,4 \\
\hline Queda do paciente & 9 & 3 & 5 & 7 & 7 & 15 & 15 & 2 & 11 & 17 & 91 & 6,7 \\
\hline Úlcera por pressão & 8 & 2 & 9 & 1 & 14 & 7 & 6 & 6 & 11 & 8 & 72 & 5,3 \\
\hline $\begin{array}{l}\text { Falta de } \\
\text { hemoderivado }\end{array}$ & 1 & 1 & 1 & 0 & 1 & 10 & 11 & 7 & 13 & 11 & 56 & 4,1 \\
\hline Evasão & 5 & 1 & 4 & 2 & 6 & 5 & 2 & 4 & 4 & 6 & 39 & 2,9 \\
\hline TOTAL & 148 & 82 & 95 & 71 & 104 & 156 & 202 & 102 & 203 & 197 & 1.360 & 100 \\
\hline
\end{tabular}

*Análise dos registros de enfermagem realizados em sete meses. 


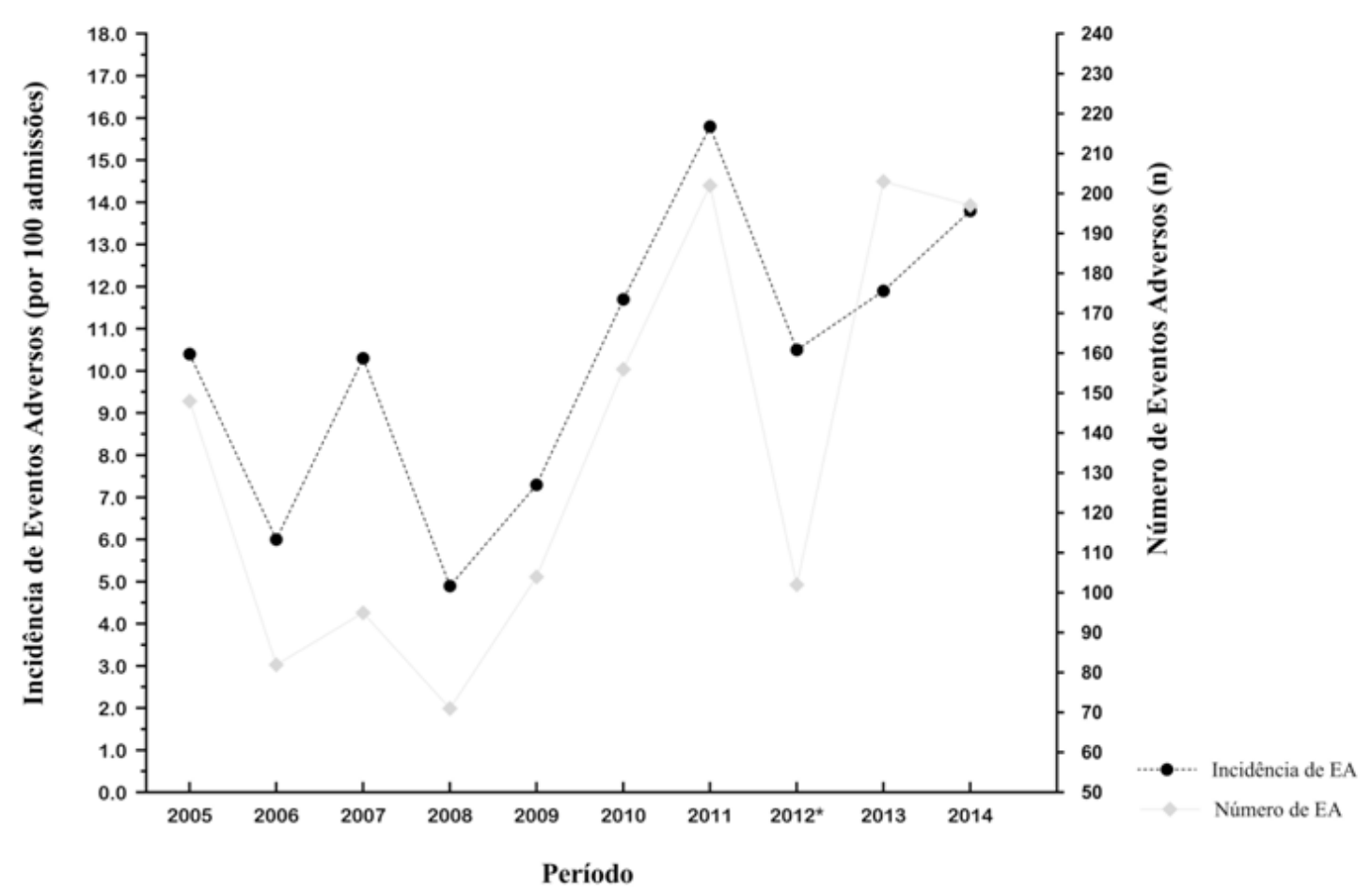

Figura 1 - Incidência de EA (por 100 pacientes) e número de EA. Jan 2005 - Dez 2014

Na figura 1 visualiza-se um decréscimo de eventos adversos em 2012, porém ressalta-se que neste ano não foram analisados os livros de agosto a dezembro. O cálculo de proporcionalidade para este ano evidencia aumento no número de EA para 175 em 12 meses, portanto, identifica-se que os registros de enfermagem continuam em um crescente a partir de 2008.

Os resultados relacionados ao evento "retirada acidental de sondas, cateteres e drenos", que foi o mais frequente, segregado por tipo de dispositivo envolvido e ano de ocorrência, estão apresentados na Tabela 2.

Tabela 2 - Distribuição dos eventos adversos do tipo "retirada acidental de sondas, cateteres e drenos" ocorridos na unidade de clínica médica de um hospital de ensino, segundo tipo e ano de ocorrência. Goiânia, GO, Brasil, 2015

\begin{tabular}{|c|c|c|c|c|c|c|c|c|c|c|c|c|}
\hline \multirow[t]{2}{*}{ Tipo de Dispositivo } & \multicolumn{10}{|c|}{ Ano } & \multicolumn{2}{|c|}{ Total } \\
\hline & 2005 & 2006 & 2007 & 2008 & 2009 & 2010 & 2011 & 2012* & 2013 & 2014 & $\mathbf{N}$ & $\%$ \\
\hline Sonda Nasoenteral & 44 & 23 & 19 & 23 & 36 & 36 & 34 & 19 & 37 & 31 & 302 & 74 \\
\hline Sonda Nasogástrica & 5 & 4 & 10 & 0 & 3 & 0 & 1 & 2 & 4 & 3 & 32 & 7,8 \\
\hline $\begin{array}{l}\text { Sonda Vesical de } \\
\text { Demora }\end{array}$ & 4 & 2 & 2 & 2 & 3 & 2 & 4 & 2 & 1 & 4 & 26 & 6,4 \\
\hline $\begin{array}{l}\text { Tubo Orotraqueal e } \\
\text { Conexões }\end{array}$ & 0 & 1 & 0 & 0 & 2 & 0 & 2 & 0 & 1 & 5 & 11 & 2,7 \\
\hline Gastrostomia & 0 & 2 & 0 & 0 & 0 & 2 & 0 & 0 & 1 & 1 & 6 & 1,5 \\
\hline Jejunostomia & 0 & 0 & 0 & 0 & 0 & 0 & 0 & 0 & 2 & 1 & 3 & 0,7 \\
\hline Sonda retal & 0 & 0 & 0 & 0 & 0 & 0 & 0 & 1 & 0 & 1 & 2 & 0,5 \\
\hline Outros** & 2 & 3 & 3 & 2 & 2 & 1 & 6 & 0 & 2 & 5 & 26 & 6,4 \\
\hline TOTAL & 55 & 35 & 34 & 27 & 46 & 41 & 47 & 24 & 48 & 51 & 408 & 100 \\
\hline
\end{tabular}

*Análise dos registros de enfermagem realizados em sete meses.

**Tubos, cateter de $\mathrm{O} 2$ e drenos 
Verifica-se na Tabela 2 que a sonda nasoenteral foi o dispositivo mais frequentemente envolvido nos casos de retirada acidental. Alguns relatos de retirada de sonda nasoenteral e nasogástrica traziam a informação de que o paciente a removeu, pois afirmava conseguir alimentar-se sozinho, o que leva a um questionamento sobre a correta indicação e uma possível utilização indiscriminada do dispositivo. Esse questionamento é reforçado pelo fato de, em alguns casos, após verificar a retirada de sonda do paciente e a alimentação por via oral, o profissional não realizou novo procedimento e aguardou discussão com a equipe sobre sua real necessidade.

No que tange ao momento da ocorrência dos eventos do tipo "retirada acidental de sondas, cateteres e drenos", identificou-se que as retiradas aconteceram no momento de movimentação do paciente, banho, deslocamento do paciente para o banheiro, durante o sono ou episódios de confusão, sem contenção.

O segundo tipo de evento adverso com maior número de casos identificados foram os erros de medicação, apresentados na Tabela 3.

Na Tabela 3, visualiza-se que o erro de omissão foi o de maior incidência (120;41,1\%), seguido dos erros de horário $(72 ; 24,6 \%)$ e de dose $(33 ; 11,3 \%)$.

Dentre os 120 erros de omissão, 50 (41,7\%) foram em detrimento de medicamentos não administrados pela enfermagem, sem relato de justificativa para o ocorrido, enquanto que $20(16,7 \%)$ foram em decorrência de atraso da entrega da prescrição, ficha, controle ou rótulo na farmácia. Destaca-se que

Tabela 3 - Distribuição dos eventos adversos do tipo "erro de medicação"ocorridos na unidade de clínica médica de um hospital de ensino, segundo tipo e ano de ocorrência. Goiânia, GO, Brasil, 2015

\begin{tabular}{|c|c|c|c|c|c|c|c|c|c|c|c|c|}
\hline \multirow[t]{2}{*}{ Tipo de Erro } & \multicolumn{10}{|c|}{ Ano } & \multicolumn{2}{|c|}{ Total } \\
\hline & 2005 & 2006 & 2007 & 2008 & 2009 & 2010 & 2011 & 2012* & 2013 & 2014 & $\mathbf{N}$ & $\%$ \\
\hline Erro de omissão & 11 & 2 & 6 & 5 & 3 & 9 & 21 & 14 & 30 & 19 & 120 & 41,1 \\
\hline Erro de horário & 4 & 2 & 2 & 1 & 0 & 2 & 8 & 4 & 23 & 26 & 72 & 24,6 \\
\hline Erro de dose & 3 & 1 & 4 & 2 & 3 & 0 & 6 & 2 & 5 & 7 & 33 & 11,3 \\
\hline $\begin{array}{l}\text { Velocidade de infusão } \\
\text { errada }\end{array}$ & 0 & 0 & 0 & 2 & 2 & 4 & 9 & 0 & 3 & 6 & 26 & 8,9 \\
\hline Troca de pacientes & 2 & 0 & 0 & 0 & 0 & 3 & 2 & 0 & 4 & 1 & 12 & 4,1 \\
\hline $\begin{array}{l}\text { Não adesão do } \\
\text { paciente ao tratamento }\end{array}$ & 0 & 0 & 0 & 0 & 0 & 2 & 2 & 0 & 3 & 1 & 8 & 2,7 \\
\hline Troca de medicação & 0 & 0 & 0 & 1 & 0 & 0 & 2 & 0 & 2 & 2 & 7 & 2,4 \\
\hline $\begin{array}{l}\text { Erro no preparo da } \\
\text { medicação }\end{array}$ & 0 & 0 & 0 & 0 & 0 & 0 & 0 & 3 & 0 & 1 & 4 & 1,4 \\
\hline Via errada & 0 & 0 & 0 & 0 & 1 & 1 & 0 & 0 & 0 & 0 & 2 & 0,7 \\
\hline Erro de prescrição & 0 & 0 & 0 & 0 & 0 & 0 & 0 & 2 & 0 & 0 & 2 & 0,7 \\
\hline $\begin{array}{l}\text { Acidente com } \\
\text { medicamento } \\
\text { disponível** }\end{array}$ & 0 & 0 & 0 & 0 & 1 & 0 & 0 & 0 & 0 & 1 & 2 & 0,7 \\
\hline $\begin{array}{l}\text { Medicamento } \\
\text { deteriorado }\end{array}$ & 0 & 0 & 0 & 0 & 0 & 0 & 0 & 1 & 0 & 0 & 1 & 0,3 \\
\hline $\begin{array}{l}\text { Medicação não } \\
\text { autorizada }\end{array}$ & 0 & 0 & 0 & 0 & 0 & 0 & 0 & 0 & 0 & 1 & 1 & 0,3 \\
\hline $\begin{array}{l}\text { Apresentação errada } \\
\text { da medicação }\end{array}$ & 0 & 0 & 0 & 0 & 1 & 0 & 0 & 0 & 0 & 0 & 1 & 0,3 \\
\hline Erro de administração & 0 & 0 & 0 & 0 & 0 & 1 & 0 & 0 & 0 & 0 & 1 & 0,3 \\
\hline TOTAL & 20 & 5 & 12 & 11 & 11 & 22 & 50 & 26 & 70 & 65 & 292 & 100 \\
\hline
\end{tabular}

*Análise dos registros de enfermagem realizados em sete meses.

**Medicamento disponível: medicamento ao qual o acompanhante e paciente tiveram acesso, porém sem acompanhamento da enfermagem. Ex: medicamentos deixados na cabeceira do leito ou trazidos pelo próprio paciente. 
na unidade de internação investigada há uma rotina estabelecida definindo o horário de entrega e recebimento de formulários para medicamentos controlados e antibióticos na farmácia que, se não obedecida, impede a dispensação e o preparo do medicamento.

Outras causas identificadas para este erro foram faltas do medicamento na instituição (nove; 7,5\%); não comparecimento do médico para administração de medicamento por via intratecal (quatro; 3,3\%); não dispensação do medicamento pela farmácia, devido à falta de prescrição (três; $2,5 \%$ ); não dispensação do medicamento pela farmácia, sem relato de justificativa (três; $2,5 \%$ ); ausência do paciente do leito no horário da medicação (três; $2,5 \%$ ); medicação prescrita não padronizada pelo hospital (três; $2,5 \%$ ).

Os registros evidenciaram problemas de relacionamento interpessoal entre as diferentes categorias profissionais da unidade e desconhecimento das funções dos mesmos no processo, na medida em que algumas prescrições não chegaram a ser enviadas à farmácia por desconhecimento ou porque havia desentendimento a respeito das respectivas responsabilidades.

A equipe médica contribuiu para a ocorrência das omissões de medicação, ao retirar da clínica, por tempo indeterminado, papeletas e prontuários, ou ao colocar prescrições e controles fora dos locais pactuados pela equipe multidisciplinar, impedindo que a equipe de enfermagem tivesse acesso às prescrições do dia, acarretando atrasos e omissões de cuidados de enfermagem primordiais, como a medicação.

Os erros de horário totalizaram 72 estando relacionados, em sua maioria, a atraso na dispensação do medicamento pela farmácia hospitalar (16;22,2\%); problemas com a bomba de infusão (nove; $12,5 \%$ ); temperatura inadequada dos quimioterápicos no momento da administração (sete; 9,7\%); ausência da prescrição, papeleta ou controle na clínica (cinco; 6,9\%); aprazamento errado (quatro; 5,5\%); paciente sem acesso venoso (três; 4,2\%). Em 10 relatos (13,9\%) não havia justificativa do atraso.

Os erros de horário estiveram associados ao aprazamento inadequado dos medicamentos, por uma interpretação equivocada da prescrição, ou erro de cálculo dos intervalos de tempo. O aprazamento inadequado gera gastos para a instituição, podendo acarretar sub ou superdosagem da medicação.

No que diz respeito ao atraso de administração do medicamento, foi observado frequente atraso na dispensação pela farmácia. Problemas com as bombas de infusão também foram identificados, a maioria devido ao número reduzido de máquinas e longo período de uso. Durante o período estudado a clínica recebeu novas bombas de infusão, que estiveram associadas a erros, desta vez porque a equipe não estava capacitada para manipulá-las.

A equipe de enfermagem, por vezes, no horário da administração de medicamentos, disponibilizava aqueles que são orais para que os próprios pacientes ou acompanhantes os fizessem. Erros relacionados a essa prática também foram observados, como ingestão de doses maiores do que a prescrita ou troca de medicação, por confusão com outros pacientes.

Os eventos relacionados a acesso vascular apresentaram expressiva taxa de ocorrência, e sinalizam falhas neste procedimento realizado rotineiramente pela equipe de enfermagem, e estão apresentados na Tabela 4.

Os eventos relacionados a acesso vascular de maior ocorrência foram: retirada não programada $(54 ; 34,4 \%)$ e acesso ineficaz $(48 ; 30,6 \%)$. Entretanto, danos diretos, relacionados ao evento, podem ser observados, como extravasamento de líquidos (28; 17,8\%), presença de sinais flogísticos $(14 ; 8,9 \%)$ e sangramento (nove; $5,7 \%$ ). 
Tabela 4 - Distribuição dos eventos adversos relacionados a acesso vascular ocorridos na unidade de clínica médica de um hospital de ensino, segundo tipo e ano de ocorrência. Goiânia, GO, Brasil, 2015

\begin{tabular}{lccccccccccccc} 
Tipo de Evento & \multicolumn{10}{c}{ Ano } & \multicolumn{1}{c}{ Total } \\
\cline { 2 - 15 } & $\mathbf{2 0 0 5}$ & $\mathbf{2 0 0 6}$ & $\mathbf{2 0 0 7}$ & $\mathbf{2 0 0 8}$ & $\mathbf{2 0 0 9}$ & $\mathbf{2 0 1 0}$ & $\mathbf{2 0 1 1}$ & $\mathbf{2 0 1 2}$ & $\mathbf{2 0 1 3}$ & $\mathbf{2 0 1 4}$ & $\mathbf{N}$ & $\%$ \\
\hline $\begin{array}{l}\text { Retirada não } \\
\text { programada }\end{array}$ & 17 & 6 & 7 & 2 & 0 & 8 & 1 & 1 & 2 & 10 & $\mathbf{5 4}$ & $\mathbf{3 4 , 4}$ \\
\hline Acesso ineficaz & 1 & 1 & 3 & 1 & 3 & 8 & 16 & 4 & 4 & 7 & $\mathbf{4 8}$ & $\mathbf{3 0 , 6}$ \\
\hline Extravasamento & 0 & 1 & 2 & 1 & 2 & 0 & 9 & 3 & 3 & 7 & $\mathbf{2 8}$ & $\mathbf{1 7 , 8}$ \\
\hline Sinais flogísticos & 0 & 0 & 1 & 1 & 0 & 0 & 4 & 1 & 3 & 4 & $\mathbf{1 4}$ & $\mathbf{8 , 9}$ \\
\hline Sangramento & 0 & 0 & 0 & 0 & 0 & 3 & 1 & 3 & 2 & 0 & $\mathbf{9}$ & $\mathbf{5 , 7}$ \\
\hline Obstrução & 0 & 0 & 0 & 0 & 0 & 0 & 1 & 0 & 2 & 1 & $\mathbf{4}$ & $\mathbf{2 , 5}$ \\
\hline TOTAL & $\mathbf{1 8}$ & $\mathbf{8}$ & $\mathbf{1 3}$ & $\mathbf{5}$ & $\mathbf{5}$ & $\mathbf{1 9}$ & $\mathbf{3 2}$ & $\mathbf{1 2}$ & $\mathbf{1 6}$ & $\mathbf{2 9}$ & $\mathbf{1 5 7}$ & $\mathbf{1 0 0}$
\end{tabular}

*Análise dos registros de enfermagem realizados em sete meses.

\section{- DISCUSSÃO}

Em 10 anos de registros de enfermagem, foram identificados 1360 eventos adversos, uma média de 11,3 EA por mês, com incidência de 10,2\%, em acordo com a literatura científica ${ }^{(1,9)}$, sendo que os relacionados à retirada acidental de sondas, cateteres e drenos foram os mais frequentes.

Estudo que analisou os registros de enfermagem em uma unidade de clínica cirúrgica, no período de 2005 a 2009, identificou a ocorrência de 264 EA, sendo que a retirada de sondas, cateteres e drenos também foi o evento de maior incidência ${ }^{(11)}$. A sondagem nasoenteral é um dos procedimentos mais prevalentes na assistência à saúde, sendo altamente recomendável para quadros de distúrbios crônicos e irreversíveis ${ }^{(12)}$.

Os resultados indicam que o papel do enfermeiro parece falho no que tange à adoção de medidas preventivas de retirada de sondas não programada, incluindo a orientação dos pacientes e familiares quanto à indicação do procedimento, os cuidados necessários com o dispositivo e os riscos da retirada não programada, assim como os riscos da realização de novo procedimento, como lesões nasais e orofaríngeas.

Medidas como avaliação da presença de delirum ou demência, informações à equipe e família sobre os riscos da retirada acidental, estimulação da participação da família e acompanhantes na tentativa não farmacológica de reduzir os riscos, contenção mecânica menor que 12 horas, são algumas medidas que a enfermagem pode implementar na tentativa de redução da retirada acidental de cateteres, sondas e drenos ${ }^{(13)}$.

Eventos adversos relacionados à medicação também ocorreram com alta frequência, sendo citados como eventos muito comuns no cenário de atendimento hospitalar, amplamente abordados na literatura científica devido à sua gravidade e ao risco de danos irreversíveis ou mesmo a morte. Um conjunto de fatores está ligado à sua ocorrência, cabendo destacar a complexidade do sistema de medicação, composto por fases interligadas e interdependentes, realizadas por mais de uma categoria profissional, e a falta de comunicação da equipe multidisciplinar.

No Brasil, o Protocolo de Segurança na Prescrição, Uso e Administração de Medicamentos do Ministério da Saúde elaborado em parceria com a Agência Nacional de Vigilância Sanitária (ANVISA), preconiza vários passos, incluindo os nove certos, abrangendo as formas de prescrever, dispensar e administrar a medicação, visando à redução de danos ao paciente ${ }^{(14)}$.

Cabe ressaltar que a enfermagem está envolvida desde o armazenamento e identificação dos medicamentos dispensados para a enfermaria, que devem estar sob sua guarda, até a devolução de sobras de medicamentos administrados para a farmácia, pois estoque de medicamentos nas enfermarias são fonte importante de erros de administração ${ }^{(14)}$. 
A omissão foi o principal erro de medicação detectado neste estudo, e pode estar associada à sobrecarga de trabalho da equipe de enfermagem, devido à alta taxa de absenteísmo e elevado número de atestados e faltas injustificadas observados na instituição, conforme apontou pesquisa realizada na região Centro-Oeste do Brasil ${ }^{(15)}$.

O uso de tecnologias no processo de medicação pode se tornar um fator de risco à manutenção da segurança do paciente. Esta pode estar associada à ausência ou inadequação do treinamento ou por negligência às informações recebidas, resultando desse modo em aumento das complicações na terapia do paciente, destacando a infusão automática de doses diferentes da prescrita ${ }^{(16)}$.

O acesso vascular, utilizado dentre outras coisas para administração dos medicamentos, é uma via aberta que expõe o paciente ao risco de infecção relacionada à assistência à saúde quando manipulado de maneira inadequada. Sua retirada não programada, além de aumentar o custo material para a instituição, e exigir retrabalho da equipe, submete o paciente a uma nova intervenção invasiva e dolorosa, que pode gerar danos físicos e psicológicos.

A prevenção de falhas infusionais contribui para diminuição de repetidas reinserções, que causam, além da dor, lesão da rede venosa periférica, agredindo a rede de defesas do paciente, gerando desconforto e preocupação ${ }^{(17)}$. A retirada não programada acontece, muitas vezes, devido ao estado de agitação do paciente, não contido mecanicamente ou por medicação, ou mesmo quando o paciente sai da clínica para a realização de procedimentos como exames, hemodiálise, cirurgias.

Pacientes não colaborativos também interferem em seu processo de medicação, evidenciando falhas na orientação do paciente e seus acompanhantes sobre o procedimento, pela equipe de enfermagem, a importância da não retirada, além das consequências de uma retirada de acesso vascular não programada.

Assimilar que os eventos adversos devem ser analisados e suas causas devem ser investigadas com abordagem sistêmica e multifatorial é o maior desafio a ser enfrentado pelas organizações de saúde em prol da segurança dos pacientes.

\section{CONCLUSÕES}

O estudo apontou o problema e a persistência dos eventos adversos ao longo da década, indicando a necessidade de adoção de medidas de prevenção que transformem essa realidade que retrata episódios de não qualidade no cuidado. Apontou também o aumento dos relatos de eventos adversos com o passar dos anos, indicando maior conscientização da enfermagem quanto à importância de registrar esse tipo de incidente que ocorre na assistência à saúde.

Apesar de relatórios de enfermagem não terem a finalidade de notificação de eventos adversos aos órgãos competentes, acredita-se que eles constituíram rica fonte de informação e permitiram identificar a ocorrência e seus diversos tipos.

Estudos que abordem em profundidade, cada tipo de evento adverso, suas causas e consequências, fazem-se necessários dada a complexidade destes. Análises de prontuários também devem ser realizadas para verificar se estes mesmos eventos foram registrados nos prontuários dos pacientes internados na clínica médica.

Perante as evidências deste estudo, acredita-se que medidas devem ser tomadas para bloquear a continuidade da ocorrência de danos aos pacientes que buscam assistência em instituições hospitalares.

A adoção de uma cultura de segurança do paciente se faz premente, assim como outras medidas direcionadas ao gerenciamento de riscos, à melhoria na comunicação entre a equipe multiprofissional, à instituição de protocolos organizacionais direcionados para segurança do paciente e às avaliações constantes das estruturas e dos processos de trabalho em busca de melhores resultados assistenciais.

O estudo apresentou limitações relacionadas à qualidade das informações encontradas nos registros de enfermagem, pois muitas anotações não possuíam informações completas e detalhadas sobre os eventos adversos, dificultando o aprofundamento da investigação e gerando lacunas sobre as causas e consequências dos mesmos, condutas clínicas e de gestão frente às ocorrências, entre outros. 
Registram-se aqui os agradecimentos ao Conselho Nacional de Desenvolvimento Científico e Tecnológico (CNPq) pela concessão da Bolsa de Iniciação Científica que auxiliou na realização desta pesquisa.

\section{O REFERÊNCIAS}

1. Kohn LT, Corrigan JM, Donaldson MS. To Err Is Human: Building a Safer Health System. Washington, DC: Committee on Quality of Health Care in America, Institute of Medicine: National Academy Press. [Internet] 1999 [acesso em 23 fev 2016]. Disponível: https://www.iom.edu/ /media/Files/Report\%20Files/1999/To-Err-is-Human/ To\%20Err\%20is\%20Human\%201999\%20\%20report\%20brief.pdf.

2. Sommella L, de Waure C, Ferriero AM, Biasco A, Mainelli MT, Pinnarelli L, et al. The incidence of adverse events in an Italian acute care hospital: findings of a two-stage method in a retrospective cohort study. BMC Health Services Research. [Internet] 2014; 7(14) [acesso em 24 fev 2016].

Disponível: http://dx.doi.org/10.1186/1472-6963-14-358.

3. Paranaguá TTB, Bezerra ALQ, Silva AEBC, Azevedo Filho FM. Prevalência de incidente sem dano e evento adverso em uma clínica cirúrgica. Acta paul. enferm. [Internet] 2013; 26(3) [acesso em 24 fev 2016]. Disponível: http://dx.doi.org/10.1590/S0103-21002013000300009.

4. de Paiva MCMS, de Paiva SAR, Berti HW. Eventos adversos: análise de um instrumento de notificação utilizado no gerenciamento de enfermagem. Rev. esc. enferm. USP. [Internet] 2010; 44(2) [acesso em 23 fev 2016]. Disponível: http://dx.doi.org/10.1590/S0080-62342010000200007.

5. Mendes W, Pavão ALB, Martins M, Moura MLO, Travassos C. Características de eventos adversos evitáveis em hospitais do Rio de Janeiro. Rev. Assoc. Med. Bras. [Internet] 2013; 59(5) [acesso em 28 fev 2016]. Disponível: http://dx.doi.org/10.1016/j.ramb.2013.03.002.

6. Tartaglia R, Albolino S, Bellandi T, Bianchini E, Biggeri A, Fabbro G, et al. Eventi avversi e conseguenze prevenibili: studio retrospettivo in cinque grandi ospedali italiani. Epidemiol Prev. [Internet] 2012; 36(3-4) [acesso em 23 fev 2016]. Disponível: http://www.epiprev.it/articolo_scientifico/eventi-avversi-e-conseguenze-prevenibili-studioretrospettivo-cinque-grandi-osp.

7. Donaldson LJ, Panesar SS, Darzi A. Patient-Safety-Related Hospital Deaths in England: Thematic Analysis of Incidents Reported to a National Database, 2010-2012. PLoS Med. [Internet] 2014; 11(6) [acesso em 23 fev 2016]. Disponível: http://dx.doi.org/10.1371/journal.pmed.1001667.

8. Empresa Brasileira de Serviços Hospitalares. Hospital das Clínicas (EBSERH). Universidade Federal de Goiás. Dimensionamento de serviços assistenciais e da gerência de ensino e pesquisa. [Internet] Brasília: EBSERH; 2014 [acesso em 23 fev 2016]. Disponível: http://www.ebserh.gov.br/documents/15796/181429/dimensionamento_ assistencial_ufg.pdf/fafe4c4a-c113-48d0-9571-0b314955c00a.

9. Mendes W, Martins M, Rozenfeld S, Travassos C. The assessment of adverse events in hospitals in Brazil. Int J Qual Health Care. [Internet] 2009; 21(4) [acesso em 06 jun 2016]. Disponível:

http://dx.doi.org/10.1093/intqhc/mzp022.

10. Ministério da Saúde (BR). Conselho Nacional de Saúde. Diretrizes e normas regulamentadoras da pesquisa envolvendo seres humanos. Resolução n. 466, de 12 de dezembro de 2012. Brasília; 2012.

11. Carneiro FS, Bezerra ALQ, Silva AEBC, de Souza LP, Paranaguá TTB, Branquinho NCSS. Eventos adversos na clínica cirúrgica de um hospital universitário: instrumento de avaliação da qualidade. Rev. enferm. UERJ. [Internet] 2011; 19(2) [acesso em 24 fev 2016]. Disponível: http://www.facenf.uerj.br/v19n2/v19n2a06.pdf.

12. Nogueira SCJ, de Carvalho APC, de Melo CB, de Morais EPG, Chiari BM, Gonçalves MIR. Perfil de pacientes em uso de via alternativa de alimentação internados em um hospital geral. Rev. CEFAC. [Internet] 2013; 15(1) [acesso em 24 fev 2016]. Disponível: http://dx.doi.org/10.1590/S1516-18462012005000079.

13. Pereira SRM, Coelho MJ, Mesquita AMF, Teixeira AO, Graciano SA. Causas da retirada não planejada da sonda 
de alimentação em terapia intensiva. Acta paul. enferm. [Internet] 2013; 26(4) [acesso em 24 fev 2016]. Disponível: http://dx.doi.org/10.1590/S0103-21002013000400007.

14. Ministério da Saúde (BR). Protocolo de segurança na prescrição, uso e administração de medicamentos. Protocolo integrante do Programa Nacional de Segurança do Paciente. Brasília: Ministério da Saúde, ANVISA; 2013. Anexo 03.

15. Coelho MA. Dimensionamento de profissionais de enfermagem das unidades de internação de adultos de um hospital de ensino da região Centro-oeste do Brasil [tese]. Goiânia (GO): Faculdade de Enfermagem da Universidade Federal de Goiás; 2013.

16. Holsbach LR, Kliemann Neto FJ, Holsbach N. Utilização do instrumento de identificação de conhecimentos para administração segura de medicamentos com o uso de infusão automática. Rev. Bras. Eng. Bioméd. [Internet] 2013; 29(4) [acesso em 24 fev 2016]. Disponível: http://dx.doi.org/10.4322/rbeb.2013.034.

17. Domingues GM, Morais FRRL, Ferreira Júnior MA. Tempo de permanência dos cateteres vasculares venosos periféricos e seus riscos para flebite relacionada ao sítio de inserção: uma revisão bibliográfica. Rev. Cient Link Jun. 2012; 2(2): 38-47. 